\title{
Corrective maintenance unplanned urban transport vehicles in the city of Manaus and its impact on costs
}

\author{
Edry Antonio García Cisneros ${ }^{1}$, João Evangelista Netto ${ }^{2}$, José Costa de Macêdo Neto ${ }^{3}$, Tirso Lorenzo Reyes Carvajal ${ }^{4}$ \\ Drs. ${ }^{1,2,3}$ Escuela Superior de Tecnología, Universidad del Estado de Amazonas. Ave. Darcy Vargas 1200, Parque Dez CP 69050- \\ 020. Manaus, AM, Brasil. \\ Dr. ${ }^{4}$ Instituto e Tecnologia e Educação Galileo da Amazônia (ITEGAM). Avenida Joaquim Nabuco, No . 1950. CEP: 69005-080. \\ Manaus. Amazonas. \\ E-mails: edry1961cu@gmail.com; joao_evangelista_neto@yahoo.com; jotacostaneto@gmail.com; tirsolrca@gmail.com
}

\begin{abstract}
Maintaining occupies a special place in the development of any carrier in the world and therefore is considered as a key sector. Under current conditions in which it is increasingly clear the need to reduce operating costs and maximize the performance of transportation, maintenance work can just improve such conditions. However, to be considered as an effective area which can ensure the efficiency requirements of the industry is important that this work using indicators to measure and evaluate the maintenance process. In this work the experience gained in a transport company bus in the city of Manaus, where he was made an analysis of the maintenance process in vehicles of urban public transport in the city of Manaus in an undertaking of great size by presents the assessment indicators failure rate and maintenance costs for engine, transmission and propeller bridge model VW bus $17230 \mathrm{E}$ OD, being the most used in the entity. The results show that it is these items the greater frequency of failures and therefore causing a substantial increase in maintenance costs and that corrective actions even solve the faults are not the most appropriate for the current conditions where efficiency is the watchword.
\end{abstract}

Keywords: Maintenance, transport, maintenance indicator.

\section{Mantenimiento correctivo no planeado en vehículos de transporte urbano en la ciudad de Manaos y su incidencia en los costos.}

\section{RESUMEN}

El mantenimiento ocupa un lugar especial en el desarrollo de cualquier empresa de transporte en el mundo y por eso es considerado como un sector clave. En las condiciones actuales en las que resulta cada vez más evidente la necesidad de reducir costos operativos y maximizar el rendimiento de los medios de transporte, la labor de mantenimiento permite justamente mejorar tales condiciones. Sin embargo, para ser considerada como un área efectiva que pueda asegurar los requerimientos de eficiencia de la industria es importante que esta labor utilice indicadores que permitan cuantificar y evaluar el proceso de mantenimiento. En este trabajo se presenta la experiencia desarrollada en una empresa de transporte de autobuses en la ciudad de Manaus, donde fue realizado un análisis del proceso de mantenimiento en los vehículos de transporte público urbano en la ciudad de Manaus en una empresa de gran porte mediante la evaluación de indicadores de frecuencia de fallas y costos de mantenimiento de motor, caja y puente propulsor de los ómnibus modelo VW 17230 E OD, por ser los más utilizados en dicha entidad. Los resultados muestran que son estos ítems los de mayores frecuencias de fallas y por tanto los que causan un sustantivo incremento de los costos de mantenimiento y que las acciones correctivas aunque solucionan las fallas no son las más indicadas en las condiciones actuales donde la eficiencia es la palabra de orden.

Palabras clave: soldabilidad; herramientas de calidad; proceso SMT; tablet.

\section{INTRODUCCIÓN}

En la ciudad de Manaos existen varias empresas dedicadas al servicio de transporte urbano colectivo y es posible apreciar que el número total de vehículos que componen cada una de estas es también muy variado pudiendo oscilar entre La composición de ómnibus en estas también es 5 y 7 hasta más de 200. A su vez el estado técnico de las vías por donde circulan estos medios casi nunca es el más adecuado debido al lógico envejecimiento por el paso del tiempo y también a la sobre 
Edry Antonio García Cisneros, et al / ITEGAM-JETIA Vol.02, No 06, pp.91-97. Junho, 2016.

explotación de las mismas sobre todo en los horarios llamados de pico. En este contexto el mantenimiento tiene entonces la misión de asegurar para los ómnibus las mejores condiciones técnicas posibles de forma tal que se pueda lograr la transportación colectiva en una ciudad cuya población asciende a más de dos millones de habitantes y que este tipo de transportación como el principal a falta de otros alternativos.

El gobierno local a través de la Fundación de Apoyo a la Investigación de Amazonas (FAPEAM) así como el nacional mediante el Consejo Nacional de Desarrollo Científico y Tecnológico de Brasil (CNPq) conscientes de esta situación aportan recursos para el desarrollo de investigaciones tendientes a mejorar la actividad del transporte y dentro de esta al tema del mantenimiento.

La sociedad brasilera de normas técnicas (ABNT, 1991) define al mantenimiento como el conjunto de acciones necesarias para que un ítems sea conservado o restaurado de forma tal que pueda mantener su estado técnico especifico.

Las actividades de mantenimiento tiene la finalidad de mantener el equipo en condiciones adecuadas de funcionamiento. Estas actividades se pueden clasificadas como: Mantenimiento correctivo planificado y no planificado, mantenimiento preventivo, mantenimiento predictivo, mantenimiento detectivo y ingeniería de mantenimiento [1]. Estos autores define el objetivo del mantenimiento como: el objetivo de garantizar a disponibilidad de funcionamiento de los equipos e instalaciones de forma tal que aseguren el proceso de producción o servicio con confiabilidad, seguridad preservando el medio ambiente y con costos adecuados.

En el transcurso del tiempo la actividad de mantenimiento ha ido evolucionando hacia formas cada vez superiores, sin embargo es posible afirmar que cada tipo de mantenimiento ha ido perfeccionando esta actividad y hoy el uso de uno u otro depende fundamentalmente del condicionamiento económico y técnico.

Todas las fases de mantenimiento y operación de los equipos tienen por objetivo supremo asegurar la función de los equipamientos, sistemas e instalaciones durante el transcurso de su vida útil y la no degeneración de su desempeño

El uso de indicadores tanto técnicos como económicos es una práctica habitual hoy en día en las empresas de excelencia y también es posible encontrar indicadores comunes en diferentes procesos y países.

En el caso de las empresas de transporte el mantenimiento correctivo aún es muy utilizado en muchas empresas dado fundamentalmente por las condiciones económicas como por la carencia de personal técnico con la capacitación adecuada para elevar a planos superiores esta importante actividad.

Analizando los resultados en la actividad del transporte es posible también afirmar que la actividad de mantenimiento preventivo y predictivo pueden ser consideradas entre las más adecuadas desde el punto de vista de la disminución de costos por el hecho de la disminución por anticipación de las fallas y otros problemas operacionales.

De acuerdo com [2] comentan sobre la complementariedad de estos dos tipos de mantenimientos y a la vez que con esto es posible cambiar un ítem no de acuerdo a su tiempo de uso sino debido a su estado técnico evitando con esto gastos innecesarios.

El planeamiento del mantenimiento incrementa las posibilidades de intervención justamente en el momento crítico estableciendo entonces el óptimo para la corrección de fallas que permitan eliminar después el riesgo de paradas totales por falta de mantenimiento.

En este trabajo se presenta la experiencia desarrollada en una empresa de transporte de autobuses en la ciudad de Manaus, donde fue realizado un análisis del proceso de mantenimiento en los vehículos de transporte público urbano en la ciudad de Manaus en una empresa de gran porte mediante la evaluación de indicadores de fallas totales en el periodo analizado y costos de mantenimiento de motor, caja y puente propulsor de los ómnibus modelo VW 17230 E OD, por ser los más utilizados en dicha entidad. Los resultados muestran que estas fallas son solucionadas por acciones correctivas y causan un sustantivo incremento de los costos de mantenimiento, además este proceder correctivo, aunque solucionan las fallas, no son las más indicadas en las condiciones actuales donde la eficiencia es la palabra de orden.

\section{METODOLOGÍA}

La metodología desarrollada en el trabajo para el análisis del mantenimiento en la empresa se basó en la información disponible por la empresa. Se utilizó el método de análisis y síntesis de la información disponible así como la estadística descriptiva. Los indicadores calculados muestran la real situación de la misma y posibilitan la toma de decisiones para las acciones de mejoras.

\section{Determinación de la situación problémica, campo, objeto de estudio y objetivo de la investigación.}

\section{Situación problémica:}

El departamento de mantenimiento de la empresa donde se realizó la investigación que opera una flota de vehículos destinados para la transportación colectiva de pasajeros en la ciudad de manaos del Estado de Amazonas, Brasil, no adopta un plan de mantenimiento preventivo como es recomendado por los fabricantes del parque de vehículos y crea su plan basado fundamentalmente a partir de una perspectiva basada en la intervención con el objetivo de realizar reparaciones y realizadas frecuentemente una vez ocurridas las fallas en estos vehículos lo que trae como consecuencia que estas acciones sean del tipo de respuesta correctiva aunque sea mejor acciones 
Edry Antonio García Cisneros, et al / ITEGAM-JETIA Vol.02, No 06, pp.91-97. Junho, 2016.

preventivas como es sugerido por los fabricantes de estos vehículos. Además, se pudo observar que no siempre se cuenta con la información necesaria que posibilite la adopción de otras acciones preventivas.

En la empresa no existe un banco de datos para las acciones de mantenimiento que posibilite profundizar en informaciones relacionadas con los intervalos de sustitución de todos los ítems de estos vehículos para las diferentes situaciones de operación de estos medios, además la verificación de las condiciones técnicas de estos son realizadas de forma empírica cuando los operadores o choferes inician el proceso de trabajo de estos vehículos es por ello que prima la condición correctiva que es realizada una vez detectada la falla. Los ítems donde se constató mayor frecuencias de ocurrencias de fallas fueron el motor, la caja de velocidades y el puente propulsor respectivamente.

Derivada de esta problemática se determina como campo de la investigación el proceso de mantenimiento de vehículos de la empresa y como objeto de estudio el ítem motor, el ítem caja y el ítem puente propulsor.

Como objeto de la investigación se plantea entonces la evaluación del proceso de mantenimiento del objeto de estudio mediante el uso de indicadores de mantenimiento.

\section{empresa.}

\section{Selección de los indicadores de mantenimiento de la}

Para cumplimentar el objetivo propuesto se utilizaron los siguientes indicadores:

- Fallas totales en los ítems motor, caja de velocidades y en el ítem puente propulsor.

- Costo de mantenimiento correctivo del ítem motor.

- Costo de mantenimiento correctivo del ítem de velocidades.

- Costo de mantenimiento correctivo del ítem puente propulsor y árbol cardan

\section{Colección de datos.}

Para el desarrollo de la investigación se realizó la colecta de datos en el período comprendido entre los meses de enero y octubre del año 2014. Fueron consultados los documentos disponibles por la empresa contentiva de informaciones esenciales para el estudio de la política de mantenimiento adoptada y se tomó como referencia la información suministrada sobre el historial de mantenimiento de los ómnibus, los tiempos de paradas para la reparación y los costos de estas operaciones. La investigación abarcó el estudio de estos tres ítems en los ómnibus modelo VW 17.230 por representar el 73,41\% del parque de vehículos existentes en la empresa.

\section{Procesamiento de la información.}

Para el procesamiento de la información fue utilizado el sistema Microsoft Excel por las ventajas del mismo como disponibilidad, posibilidades en el tratamiento estadístico de la información, y rapidez en el procesamiento de la información así como las posibilidades de salidas graficas e numéricas de los resultados.

\section{RESULTADOS}

\section{Análisis del indicador fallas totales en los tres ítems} en el período evaluado.

En posición de los datos colectados en los registros de mantenimiento en el período de enero a octubre de 2014 fue posible realizar el levantamiento del indicador número total de fallas en este período para los ítems motor, caja de velocidades y puente propulsor (Gráfico 1).

La gráfica 1 muestra que los mayores valores en las fallas totales en el período corresponden a los ítems motor y caja de velocidades con 163 y 160 fallas respectivamente mientras que el menor valor fue en el ítem puente propulsor con 55 fallas.

Gráfico 1 - Fallas totales de los tres ítems en el período evaluado

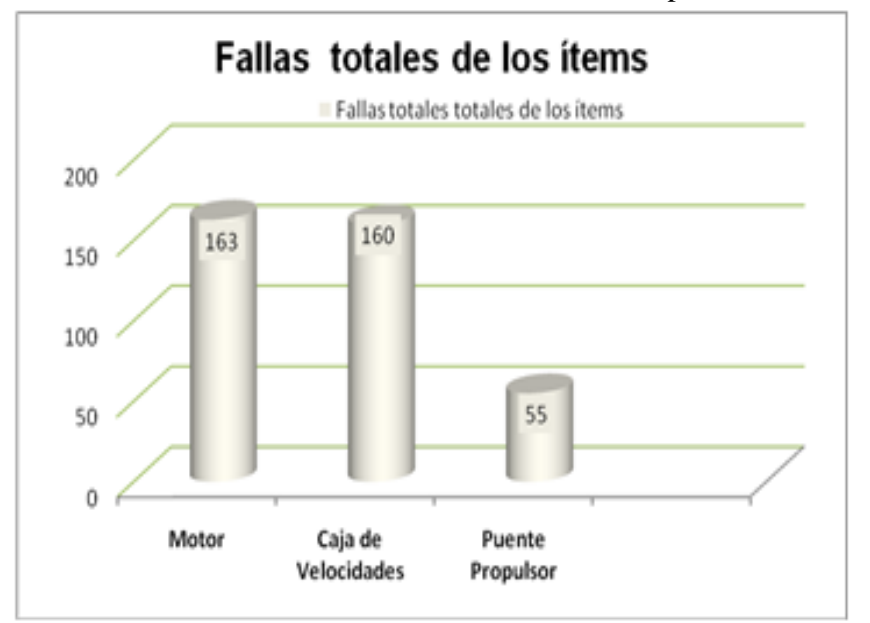

Fuente: Autores, (2016).

Los principales problemas relacionados con las fallas del ítem motor diesel MWM según el levantamiento de datos realizado están relacionadas con bajo niveles de aceite en el motor por fugas en las tuberías, fallas en las bombas de aceite, obstrucciones en los orificios de captura del aceite en el Carter y pérdida de presión en el bombeo a los cojinetes por excesivas holguras en estos y también en las bielas. Estas fallas sugieren falta de sistematicidad en las labores de mantenimiento.

En la caja de velocidades las principales fallas están asociadas a desgastes en los engranes de las marchas centradas fundamentalmente en la tercera y cuarta marcha.

En el estudio del puente propulsor las principales fallas están asociadas también a desgastes tanto en la corona del diferencial como en el piñón de ataque del mismo. 
Edry Antonio García Cisneros, et al / ITEGAM-JETIA Vol.02, Nº6, pp.91-97. Junho, 2016.

Las probables causas de estas fallas pueden relacionarse a problemas de operación de los ómnibus y al proceso de envejecimiento de esta técnica.

Estos ómnibus son utilizados en la ciudad donde las condiciones viales no son óptimas, la calidad técnica de las vías no son las mejores en la ciudad por lo que es posible apreciar la existencia de insuficiencias como estrechez de las calles, irregularidades y obstrucciones en las vías de transportación dados por el envejecimiento de la ciudad y la falta de mantenimiento de la red vial.

También es posible relacionar el sobre uso de los ómnibus en los llamados horarios picos de la ciudad donde ocurre el mayor tránsito (entre las $7.00 \mathrm{am}$ y $9.00 \mathrm{am}$; las $11.00 \mathrm{am}$ y las 15.00 horas y entre las 17.00 y 20.00 horas) y que corresponden también a los períodos de mayor aglomeración de la población y por consiguiente de mayor nivel de ocupación de las capacidades de carga de estos vehículos que en ocasiones llegan a sobre uso de estas capacidades.

Además de estos factores se puede plantear también las insuficiencias en la sistematicidad y selección adecuada del tipo de actividad de mantenimiento en la empresa así como el envejecimiento de los ómnibus.

\section{Análisis de los costos del mantenimiento correctivo realizado en el ítem motor.}

Cuando existen fallas en los motores se produce entonces un incremento de los costos pues esto implica un mayor tiempo de inactividad debido a la realización del mantenimiento correctivo que conlleva al necesario proceso de inspección, desarme, cambio del ítem o reparación de una parte de este (que generalmente es una actividad compleja que puede incluir desde la rectificación del block, el desarme del conjunto pistón cilindro aros, etc hasta la sustitución de una parte de este conjunto o el conjunto completo como sucedería por ejemplo en el caso de que fuera los inyectores y sus agujas.

En el caso de la mencionada empresa además de las complejidades propias de esta actividad muchas veces las piezas necesarias no se encontraban disponibles en el mercado local lo que provocó mayor tiempo en la actividad de gestión y compra de la misma en otros mercados del país. Como resultado el plazo de espera para reparar este ítem según documentos revisados y constatado por los propios mecánicos fue de 120 días.

Los valores de los costos de las piezas utilizadas en el mantenimiento correctivo del ítem motor son mostrados en la tabla a seguir:

Tabla 1. Costos de mtto correctivo en ítem motor.

\begin{tabular}{|c|c|c|c|}
\hline \multicolumn{4}{|c|}{ Valores en piezas usadas en el mantenimiento correctivo } \\
\hline Ítem Motor & $\begin{array}{c}\text { Canti } \\
\text { dad }\end{array}$ & $\begin{array}{c}\text { Valor } \\
\text { Unitario, } \\
\text { R\$ }\end{array}$ & $\begin{array}{c}\text { Valor, } \\
\text { R\$ }\end{array}$ \\
\hline Rectificación del block & 1 & 2585,25 & 2585,25 \\
\hline
\end{tabular}

\begin{tabular}{|c|c|c|c|}
\hline $\begin{array}{l}\text { Camisa del motor MWM } \\
\text { D }-229 / 17.230\end{array}$ & 6 & 137,72 & 826,32 \\
\hline $\begin{array}{lrll}\text { Kit pistón } & \text { MWM } & \text { D- } \\
229 / 17.230 & & \end{array}$ & 6 & 110,90 & 665,40 \\
\hline $\begin{array}{l}\text { Biela motor MWM } 6.12 \\
\text { VWO }\end{array}$ & 6 & 294,05 & 1764,30 \\
\hline $\begin{array}{l}\text { Metales del Cojinete de } \\
\text { deslizamiento del apoyo }\end{array}$ & 12 & 41,78 & 501,36 \\
\hline $\begin{array}{l}\text { Metales del Cojinete de } \\
\text { deslizamiento de la biela }\end{array}$ & 12 & 22,53 & 270,36 \\
\hline $\begin{array}{c}\text { Kit aros MWM } 6.12 \\
17.230\end{array}$ & 1 & 84,27 & 84,27 \\
\hline $\begin{array}{l}\text { Metales de las bielas } \\
\text { MWM } 6.1217 .230\end{array}$ & 6 & 19,40 & 116,40 \\
\hline $\begin{array}{r}\text { Arandela de presión } \\
\text { MWM } 6.12 \text { / AE } 112 \mathrm{P}- \\
0.25\end{array}$ & 1 & 29,76 & 29,76 \\
\hline Válvula de admisión & 6 & 19,77 & 118,62 \\
\hline Válvula de escape & 6 & 37,95 & 227,70 \\
\hline $\begin{array}{ll}\text { Guía de la válvula de } \\
\text { admisión } \\
\text { 230 }\end{array}$ & 6 & 10,60 & 63,60 \\
\hline $\begin{array}{l}\text { Guía de la válvula de } \\
\text { escape MWM 6.12/D 230 }\end{array}$ & 6 & 13,85 & 83,10 \\
\hline $\begin{array}{l}\text { Empujador de la válvula } \\
\text { MWM 6.12/T / TCA } 6.12 \\
17.230\end{array}$ & 12 & 16,42 & 197,04 \\
\hline $\begin{array}{l}\text { Junta de la culata MWM D } \\
\text { 230/6.12 SCEURO III }\end{array}$ & 6 & 36,88 & 221,28 \\
\hline $\begin{array}{l}\text { Tubo de combustible } \\
\text { VWO } 17.230 \text { EOD filtro }\end{array}$ & 2 & 60,58 & 121,16 \\
\hline $\begin{array}{l}\text { Tubo lubricante } 962 \text { / PSL } \\
\text {-962/OC60 filtro }\end{array}$ & 1 & 21,50 & 21,50 \\
\hline \multicolumn{3}{|l|}{ Total R\$ } & 7932,77 \\
\hline
\end{tabular}

Fuente: Autores, (2016).

Partiendo de los costos de las piezas utilizadas para el mantenimiento correctivo del ítem motor ( $\$$ \$ 7932,77) y del tiempo medio de parada para la solución de los fallos (120 días) es posible entonces determinar el valor medio dejado de recaudar en el período de parada para la reparación por la falla del motor diesel del vehículo de pasajeros.

Tabla 2. Valor medio de dejado de recaudar en el período ítem motor.

\begin{tabular}{|l|r|}
\hline \multicolumn{1}{c|}{ Indicador } & \multicolumn{1}{c|}{ Valor, R\$ } \\
\hline $\begin{array}{l}\text { Días de espera por carro parado por } \\
\text { reparación del ítem motor }\end{array}$ & 120 días \\
\hline Cantidad media diarias de pasajes & 1428,00 \\
\hline Valor unitario de cada pasaje en ómnibus & 2,75 \\
\hline Valor medio diario de recaudación & 3927,00 \\
\hline $\begin{array}{l}\text { Valor medio dejado de recaudar por } \\
\text { concepto de parada del ómnibus para } \\
\text { reparar motor }\end{array}$ & $\mathbf{4 7 1} \mathbf{2 4 0 , 0 0}$ \\
\hline
\end{tabular}

Fuente: Autores, (2016). 
Edry Antonio García Cisneros, et al / ITEGAM-JETIA Vol.02, No 06, pp.91-97. Junho, 2016.

Calculando entonces el valor dejado de recaudar debido a una falla del ítem motor de un vehículo que exige una intervención correctiva no planeada con una duración media de 120 días implica una perdida financiera de media $\mathrm{R} \$ 471240,00$. Como se aprecia es un valor significativo. realizado en el ítem caja de velocidades.

Del mismo modo los costos relacionados a las fallas en la caja de velocidades que tienen como causa principal el bajo nivel de aceite lubricante, la falta de mantenimiento a la hora debida (sobre uso del lubricante) así como la utilización de kit del embrague no apropiado con la caja de velocidades para este tipo de vehículo, así como el uso de piezas con baja calidad técnica conllevan a la compra y substitución de varias piezas como se aprecia en la tabla 3 .

Tabla 3 - Costos relacionados a las fallas en ítem caja de velocidades.

\begin{tabular}{|c|c|c|c|c|}
\hline \multicolumn{5}{|c|}{ Defectos derivados de la falta de mantenimiento preventivo } \\
\hline \multirow{4}{*}{$\begin{array}{l}\text { Caja de cambio } \\
\text { de velocidades }\end{array}$} & \multicolumn{4}{|c|}{ Bajo nivel de aceite lubricante } \\
\hline & \multicolumn{4}{|c|}{ Sobre uso del lubricante } \\
\hline & \multicolumn{4}{|c|}{$\begin{array}{l}\text { Utilización de kit de embrague incompatible con la } \\
\text { caja de cambio }\end{array}$} \\
\hline & \multicolumn{4}{|c|}{ Utilización de piezas de baja calidad } \\
\hline \multicolumn{5}{|c|}{ Valores de las piezas utilizadas en el mantenimiento correctivo } \\
\hline \multicolumn{2}{|c|}{ Ítem Caja de velocidades } & है & $\begin{array}{c}\text { Valor } \\
\text { Unitari } \\
\text { o, R\$ }\end{array}$ & Valor, $\mathbf{R} \$$ \\
\hline \multicolumn{2}{|c|}{$\begin{array}{l}\text { Eje piloto de cambio FSD } \\
6406\end{array}$} & 1 & 776,98 & 776,98 \\
\hline \multicolumn{2}{|c|}{ Cubierta sincronizador } & 1 & 161,02 & 161,02 \\
\hline \multicolumn{2}{|c|}{$\begin{array}{l}\text { ngranaje móvil 3ra marcha } \\
\text { FSB 6406A }\end{array}$} & 1 & 518,73 & 518,73 \\
\hline \multicolumn{2}{|c|}{$\begin{array}{l}\text { Engranaje fijo 3ra marcha } \\
\text { FSB } 6206^{\mathrm{a}} \mathrm{VWO}\end{array}$} & 1 & 623,86 & 623,86 \\
\hline \multicolumn{2}{|c|}{$\begin{array}{l}\text { Engranaje móvil 4ta marcha } \\
3003234 \text { VWO FSB 6406A }\end{array}$} & 1 & 272,55 & 272,55 \\
\hline \multicolumn{2}{|c|}{$\begin{array}{l}\text { Engranaje fijo 4ta marcha } \\
\text { FSB 6406A VWO }\end{array}$} & 1 & 367,63 & 367,63 \\
\hline \multicolumn{2}{|c|}{ Buje del eje del tenedor VWO } & 10 & 8,51 & 85,10 \\
\hline \multicolumn{2}{|c|}{$\begin{array}{lllr}\text { Sincronizador } & 3 \mathrm{ra} & \text { e } & 4 \mathrm{ta} \\
\text { marchas VWO } & 2 \mathrm{RK} & 311319 \\
\text { FSB 5405 A } & & \\
\end{array}$} & 1 & 749,00 & 749,00 \\
\hline \multicolumn{4}{|l|}{ Total, R\$ } & 3554,87 \\
\hline
\end{tabular}

Fuente: Autores, (2016).

Basado en los costos de adquisición de esas piezas para el mantenimiento correctivo en ítem caja de velocidades ( $\mathrm{R} \$$ 3554,87 y del tiempo medio de espera para la reparación (60 días) fue posible determinar el valor medio dejado de recaudar en el periodo de parada por fallas de este ítem. Los valores son mostrados en la tabla 4.
Tabla 4 - Valor medio dejado de recaudar en el periodo ítem caja de velocidades

\begin{tabular}{l|r}
\hline Denominación & Valor, R\$ \\
\hline $\begin{array}{l}\text { Cantidad de días parados por espera para } \\
\text { reparación }\end{array}$ & 60 \\
\hline Número medio de pasajeros por día & 1428 \\
\hline Valor unitario de cada pasaje & $\mathrm{R} 2,75$ \\
\hline $\begin{array}{l}\text { Valor medio dejado de recaudar } \\
\text { durante el periodo de parada por } \\
\text { reparación }\end{array}$ & $\mathbf{R} \mathbf{2 3 5} \mathbf{6 2 0 , 0 0}$ \\
\end{tabular}

Fuente: Autores, (2016).

Por consiguiente las fallas ocurridas en el periodo analizado en el ítem caja de velocidades incidieron también en importantes pérdidas de recaudación en los 60 días de paradas para reparación alcanzando un valor ascendiente a $\mathrm{R} \$ 235620,00$.

Costos de mantenimiento correctivo del ítem Puente propulsor: Usando la misma metodología, se procedió a la determinación de los costos derivados de la adquisición de piezas para la reposición por fallas y/o problemas técnicos en el ítem puente propulsor de los ómnibus, fue constatado como las fallas más frecuentes: Falla de la cruceta del árbol cardan, así como salideros de aceite del puente propulsor por las ruedas traseras y ruido en el núcleo del diferencial del puente propulsor (este ruido evidentemente se relaciona a ajustes y regulaciones entre el piñón de ataque y la corona del diferencial).

Tabla 5. Costos relacionados a las fallas en ítem Puente Propulsor y Árbol Cardan.

\begin{tabular}{|c|c|c|c|c|}
\hline \multirow{3}{*}{$\begin{array}{l}\text { ítem puente } \\
\text { propulsor }\end{array}$} & \multicolumn{4}{|c|}{ Rotura de la junta homocinética } \\
\hline & \multicolumn{4}{|c|}{ Ruido en el diferencial } \\
\hline & \multicolumn{4}{|c|}{$\begin{array}{l}\text { Salideros de aceite en el puente propulsor por las } \\
\text { ruedas traseras }\end{array}$} \\
\hline \multicolumn{5}{|c|}{ Valores de las piezas utilizadas en el mantenimiento correctivo } \\
\hline \multicolumn{2}{|c|}{ Ítem Puente propulsor } & 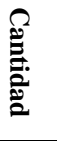 & $\begin{array}{c}\text { Valor } \\
\text { Unitario, } \\
\text { R\$ }\end{array}$ & Valor, $\mathbf{R} \$$ \\
\hline \multicolumn{2}{|c|}{ Cojinete de rodamiento VWO } & 2 & 67,59 & 135,18 \\
\hline \multicolumn{2}{|c|}{ Tornillo cardan VWO / dedos $3 / 8$} & 20 & 1,47 & 29,40 \\
\hline \multicolumn{2}{|c|}{$\begin{array}{l}\text { Cruceta del árbol cardan } \\
\text { OF1721/VWO17.2 }\end{array}$} & 4 & 41,00 & 164,00 \\
\hline \multicolumn{2}{|c|}{ Forro protector del árbol cardan } & 1 & 180,00 & 180,00 \\
\hline \multicolumn{2}{|c|}{$\begin{array}{l}\text { Terminal Yoke central } \\
\text { VWO17210-349 }\end{array}$} & 2 & 109,09 & 218,18 \\
\hline \multicolumn{2}{|c|}{$\begin{array}{l}\text { Conjunto piñón y corona del } \\
\text { diferencial }\end{array}$} & 1 & 1830,90 & 1830,90 \\
\hline \multicolumn{2}{|l|}{ Caja de satélites } & 1 & 2088,22 & 2088,22 \\
\hline \multicolumn{4}{|l|}{ Total R\$ } & 4645,88 \\
\hline
\end{tabular}

Fuente: Autores, (2016). 
Edry Antonio García Cisneros, et al / ITEGAM-JETIA Vol.02, No 06, pp.91-97. Junho, 2016.

Basándonos entonces en los costos de adquisición de piezas para el mantenimiento correctivo del ítem puente propulsor y árbol cardan que ascendió en el período analizado a 4 645,88 y del tiempo medio de espera para reparación (15 días) fue calculado el valor medio dejado de recaudar en el período de esta parada por los problemas anteriormente mencionados. Los resultados son mostrados en la tabla 6.

Tabla 6. Valor medio dejado de recaudar en el periodo ítem puente propulsor y árbol cardan.

\begin{tabular}{|l|c|}
\hline Denominación & Valor , R\$ \\
\hline $\begin{array}{l}\text { Valor medio dejado de recaudar durante } \\
\text { el periodo de parada por reparación }\end{array}$ & 15 \\
\hline Número medio de pasajeros por día & 1428 \\
\hline Valor unitario de cada pasaje & $\mathrm{R} \$ 2,75$ \\
\hline Totales, R\$ & $\mathbf{R} \$ \mathbf{5 8 9 0 5 , 0 0}$ \\
\hline
\end{tabular}

Fuente: Autores, (2016).

Por tanto esas fallas o problemas técnicos en el puente y árbol cardan de los ómnibus analizados en el periodo e la investigación acarrean igualmente pérdidas significativas pues resulta que el tiempo medio es considerado elevado (15 días) de inactividad productiva lo que representa por concepto de dinero dejado de recaudar un valor ascendente a $\mathrm{R} \$ 58$ 905,00.

Grafico 2. Costos y pedidas de recaudación por fallas en los tres ítems analizados.

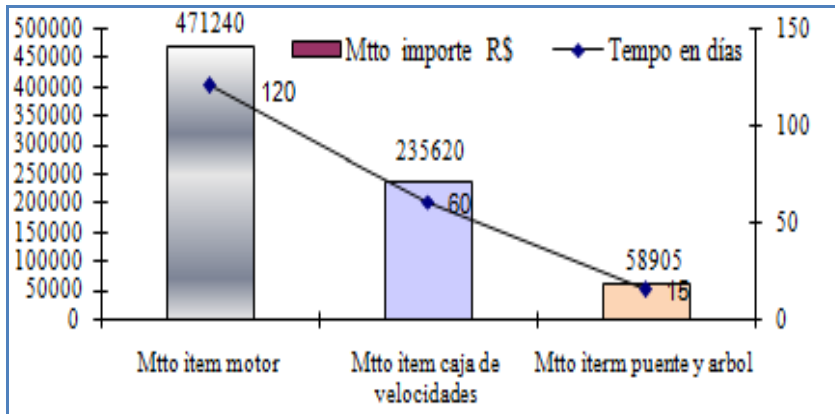

Fuente: Autores, (2016).

Como es mostrado en el grafico 2 , los costos y perdidas por ómnibus parados por causas asociadas a mantenimiento correctivo no programados resultaron muy elevados en los casos de los motores Diesel, esto es comprensible pues este ítem es de los tres analizados el más complejo por la cantidad de piezas y sistemas mecánicos componentes y por lo tanto demanda más tiempo de servicio tanto para el proceso de desarme, el de defectación como también para los procesos de reparación y substitución de las piezas y o conjuntos y sistemas con fallas que resultaron irreparables, todo este implicó un tiempo de paradas considerado muy elevado(120 días) si es comparado con los tiempos de reparación de los restantes ítem analizados.
A partir del análisis realizado con relación al tipo de mantenimiento adoptado ( en este caso correctivo no planificado) y de las repercusiones en términos de costos y perdidas de dinero dejado de recaudar para esta empresa por la forma de respuesta que esta adoptó ante los problemas técnicos y fallas surgidas en los tres ítem analizados fue posible obtener informaciones esenciales para el desarrollo de un plan de acciones preventivas las cuales podrían en el futuro reducir significativamente estos costos y perdidas lo que mejoraría el desempeño operacional de estos vehículos.

\section{CONCLUSIONES}

Los ítems que sistemáticamente causan fallas significativas en el rendimiento operacional de los ómnibus de transporte colectivo en al ciudad y que provocan un incremento en los costos de mtto y perdidas por concepto de dinero dejado de recaudar por paradas para reparación resultaron ser: Motor, caja de velocidades y puente propulsor y árbol cardan respectivamente.

Fue demostrado que las acciones correctivas pueden resolver el problema en el momento de ocurrencia de la falla pero tienen una incidencia negativa por el hecho de que al no estar planeada produce el mencionado incremento de los costos debido a que no siempre se cuenta con las piezas de repuesto en almacén.

En los costos totales por conceptos de mantenimiento inciden también otros factores que van desde el propio estado técnico de los ómnibus, el clima, el estado técnico de las vías de transportación, los horarios de trabajo hasta las habilidades y conocimientos de los choferes por lo que las acciones encaminadas a resolver estos problemas deben tener un carácter integrador.

\section{AGRADECIMIENTOS}

Especial agradecimiento o a la Fundación de apoyo a la investigación del estado de Amazonas (FAPEAM) así como al Consejo Nacional de Investigación y Desarrollo de Brasil (CNPq) por su apoyo técnico y financiero en el desarrollo de esta investigación. A los directivos y obreros de la empresa de ómnibus urbano de la ciudad de Manaos donde se desarrolló la investigación por su apoyo durante todo el período de la investigación. A la dirección de la EST y de la UEA por su apoyo en la investigación.

\section{RECOMENDACIONES}

Realizar un planeamiento objetivo de los proceso de mantenimiento que consideren estas valoraciones y que incluyan también acciones de capacitación no solo a operarios sino a todo el personal técnico de la empresa.

\section{BIBLIOGRAFÍA}

[1] PINTO, A. K., XAVIER, J. N. Mantenimiento: Función estratégica. Río de Janeiro. Qualitymark, 2001. 
[2] NAVEGA, M. B. J.; DAUMAS JÚNIOR, O. E. F. Mantenimiento Predictivo en motores. Bolsista de Valor: Revista de divulgación del Proyecto Universidad Petrobras y IF Fluminense, v. 2, n. 1, p. 173-177, 2012.

[3] DIAS, A.; CASTRO, J.R.P.; FARINA, Everton; MELGAR, Oscar Bernardo Ancieta; Kagueiama, H.A. Análise de operação e manutenção de frota de ônibus para redução do consumo de diesel em empresas de transporte coletivo. 2007.

[4] DiAS, A., CASTRO J.R.P., MATOS, F.F. de C., Projeto e implementação da manutenção em frotas: um relato de experiência, Revista Mantener del Comite Panamericano de Ingenieria del Mantenimiento da la UPADIwww.mantenimientomundial.com. ano 3, n.10,p.37-49, Buenos Aires, Argentina, setembro, 2002.

[5] DIRECTORATE-GENERAL ENERGY AND TRANSPOT, Transport, disponível emhttp://ec.europa.eu/transport/index_en.html, acessado em julho 2007.

[6] ECODRIVEN, Evaluation of Eco Driving. Disponível em http://www.ecodrive.org, acessado em julho 2014.

[7] ECO-DRIVING EUROPE. Disponível em http://www.ecodrive.at, acessado julho 2014.

[8] FUEL ECONOMY.Energy Efficiency.Disponível em http://www.fueleconomy.gov/,acessado em julho 2007.

[9] IDAE. Guía para la Gestión del Combustible en las Flotas de Transporte por Carretera. Madrid, Janeiro de 2006, Disponível em <http://www.idae.es/>, Acessado em Novembro 2014.

[10] OliVeIRA, C. A. de; ROSA, A. da. Motores de combustión interna-alcohol y gasolina. Santa María: CEP SENAI, 2008.

[11] LEANDRO, M. J.; GRZESZEZESZYN, G. Gestión de costos indirectos - costos de mantenimiento industrial. Revista Electrónica Lato Sensu, 2008. 